THORNDIKE, E. L., \& LORGE, I. The teacher's word book of 30,000 words. New York: Bureau of Publications, Teachers College, Columbia University, 1944.

TULVING, E. Subjective organization in free recall of "unrelated" words. Psychological Review, 1962, 69, 344-354.

TULVING, E. Theoretical issues in free recall. In T. R. Dixon and D. L. Horton (Eds.), Verbal behavior and general behavior theory. Englewood Cliffs, N.J: Prentice-Hall, 1968. Pp. 2-36.

\section{NOTES}

1. The computation of bidirectional organizational units, i.e., disregarding the order in which the members of an organizational unit occur is a modification of Bousfield \& Bousfield's (1966) measure introduced by Gorfein, Blair, \& Rowland (1968).

2. The formula as originally presented was:

$$
\frac{\text { Observed }}{2(C-1)}-\frac{C}{h k}
$$

However, Arjan K. Jhangiani has pointed out that the derivation of this formula should lead to the present formulation, i.e.,

$$
\frac{\text { Observed }}{\mathrm{C}-1}-\frac{2 \mathrm{C}}{\mathrm{hk}} \text {. }
$$

\title{
Increase in incentive amount with verbal reinforcement*
}

\section{LAWRENCE WEINSTEIN, $\nmid$ University of Melbourne, Melbourne, Australia and \\ VINCENT M. COLUCCI, University of Maine, Portland, Maine 04103}

An increase in the amount of verbal reinforcement resulted in gradual, as opposed to sudden, increases in behavior and no evidence of positive incentive contrast effects. These results with human Ss agree with the data obtained from animal Ss and suggest that a shift in incentive size in humans affects learning as opposed to some more temporary mechanism.

Although reward decrements often produce negative incentive contrast effects in which the performance of the shifted Ss drops significantly below the level of the low-reward control group (e.g., Crespi, 1942; Weinstein, 1970), reward increments usually do not produce positive incentive contrast effects in which the performance of the shifted group rises significantly above the level of the high-reward control group (e.g., Ashida \& Birch, 1964; Collier, Knarr, \& Marx, 1961). While most studies have investigated the effects of shifting incentive magnitude with infrahuman organisms, few experiments have used human Ss. The present study attempted to determine how human $S$ s respond to an increase in incentive size.

Initially, in a pilot study it was determined that telling an $S$ immediately after his first answer to mental multiplication problems that he had received 7 points, 3 points, or 1 point results in high, intermediate, and low levels of performance, respectively. In the present study larger and smaller rewards are defined in terms of whether they

*We thank Paul Lee for running Ss.

$\dagger$ Requests for reprints should be sent to Lawrence Weinstein, Department of Psychology, University of Melbourne, Parkville, Victoria 3052, Australia. produce higher or lower levels of performance, respectively. SUBJECTS

The Ss were 13 male and 11 female undergraduate students enrolled in an introductory psychology course at the University of Maine at Portland. The Ss were assigned randomly to each of four equal groups.

\section{MATERIALS}

The materials consisted of a Kodak Carousel 750 -slide projector, $51 / 2 \mathrm{ft}$ from a $5 \frac{1}{2}$-in.-square piece of gray metal that served as a screen, $2 \times 1 \frac{1}{2}$ in. slides with digits typed on them (i.e., $776 \times 7$ ), and a stopwatch.

\section{PROCEDURE}

Each $\mathrm{S}$ worked a different sequence of the same 15 mental multiplication problems. One answer or $30 \mathrm{sec}$ (whichever came first )was allowed for each problem and there were $8 \mathrm{sec}$ between problems.

Table 1 summarizes the experimental procedure.

The problems were worked in four situations. Some Ss received no reinforcement after their answers (N). Other Ss received a high $(\mathrm{H})$ reward, 7 points, after answering the $1 \mathrm{st}$, 2nd, 4th, 6 th, 9th, 10th, 11th, 12th, and 14th problems, and, finally, some individuals received a low (LS) reward, 1 point, or a medium (MS) reward, 3 points, through Problem 11 and then experienced a shift in reinforcement magnitude to $\mathrm{H}$ after the 11 th problem.

Ss who received LS, MS, or $\mathrm{H}$ were read the following instructions: "This is an experiment in abstract problem solving, the ability to rapidly work problems involving abstract reasoning. You will be given some problems to work. Each one consists of a three-digit number multiplied by a one-digit number. You are to mentally, without pencil and paper, multiply the numbers as quickly as you can and then tell me your answer. You will receive from 1 to 10 points after each answer; the speed and accuracy with which you answer will earn you more points. You will be told periodically how you are doing." In the instructions to the $\mathrm{N}$ Ss, reference to receiving points was omitted. RESULTS

Latency means (the time between slide onset and the first response) were examined in the analysis of the results.

From Fig. 1 it appears that for Problems 1-11 the H Ss took less time to answer than did the LS or MS Ss, the MS individuals took less time to answer than the LS Ss, and, finally, the $\mathrm{N}$ group took longer to answer than the LS group.

The mean latency per problem from Problems 1-11 differed significantly among the four groups by analysis of variance $[F(3,20)=3.19, p<.05]$

By Mann-Whitney U tests the difference between $\mathrm{H}$ and LS, $\mathrm{H}$ and MS, LS and MS, and $\mathrm{LS}$ and $\mathrm{N}$ was each statistically significant $(p<.05)$.

Figure 1 indicates that for Problems 12-15, the MS and LS groups each took longer to answer than the $\mathrm{H} \mathrm{Ss}$. By Mann-Whitney $U$ tests, the mean latency per problem from Problems 12-15 differed significantly $(\mathrm{p}<.05)$ between $\mathrm{H}$ and $L S$, and $H$ and $M S$.

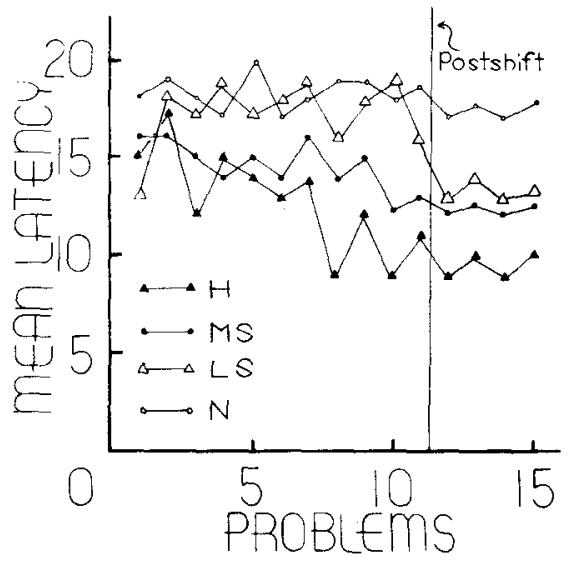

Fig. 1. Mean latency in seconds per problem. Groups LS and MS shifted after Problem 11 denoted by arrow. 


\section{DISCUSSION}

The findings that for Problems 1-11 (1) the $\mathrm{N}$ group took significantly more time to answer than the LS group, and that (2) the H Ss took significantly less time to respond than the LS individuals indicate that: (1) saying " 1 " after an answer served as a reinforcing event, where a reinforcement is defined as an event that produces a significantly higher level of performance as compared to a control group that does not experience the event, and (2) three discriminably different levels of reinforcement were used, where different amounts of reward are defined in terms of whether or not they produce significantly different levels of performance.

The experiment failed to demonstrate positive incentive contrast effects with an increase in the amount of reinforcement. This result is consistent with most studies that have increased incentive magnitude with animal Ss (e.g., Ashida \& Birch, 1964; Collier et al, 1961).

The findings that for Problems 12-15 the LS and MS groups each took significantly longer to iespord than the $\mathrm{H}$ individuals is in accord with many recent investigations with animals where decrements in the concentration of sucrose solutions do not lead to rapid performance decrements (e.g., Homzie \& Ross, 1962; Ison \& Glass, 1969; Rosen \& Ison, 1965). These gradual decreases in performance suggest that, under some yet unspecified conditions, a reduction in incentive size with animals affects learning as distinct from motivational or performance variables. The gradual change in behavior in the present experiment suggests that an increase in reward magnitude with humans results in a change in some permanent excitatory mechanism as distinct from a more transitory motivational or performance factor.

It would appear that human Ss respond to an increase in reward magnitude as do some infrahuman organisms.

$$
\text { REFERENCES }
$$

ASHIDA, S., \& BIRCH, D. The effects of incentive shift as a function of training. Psychonomic Science, 1964, 1, 201-202.

COLLIER, G., KNARR, F. A., \& MARX, M. H. Some relations between the intensive
Table 1

Summary of Experimental Procedure: Amount of Reward Received for Each Phase and Group

\begin{tabular}{lcccc}
\hline & N & LS & MS & H \\
\hline Preshift & - & 1 & 3 & 7 \\
Postshift & - & 7 & 7 & 7 \\
\hline
\end{tabular}

properties of the consummatory response and reinforcement. Journal of Experimental Psychology, 1961, 62, 484-495.

CRESPI, L. P. Quantitative variations of incentive and performance in the white rat. American Journal of Psychology, 1942, 55, 467-517.

HOMZIE, M. J., \& ROSS, L. E. Runway performance following a reduction in the concentration of a liquid reward. Journal of Comparative \& Physiological Psychology, $1962,65,1029-1033$.

ISON, J. R., \& GLASS, D. H. "Classical" versus "instrumental" exposure to sucrose rewards and later instrumental behavior following a shift in incentive value. Journal of Experimental Psychology, 1969, 79, 582-583.

ROSEN, A. J., \& ISON, J. R. Runway performance following changes in sucrose rewards. Psychonomic Science, 1965, 2 , 335-336.

WEINSTEIN, $L$. Negative incentive contrast with sucrose. Psychonomic Science, 1970, 19, 1314.

\section{ERRATUM}

THORSHEIM, H. I. NLM strength and CVC-CVC response-time recall.-Psychon. Sci., 1970, 20 (2), 101-102. The title should read as follows: NLM strength and response-term recall. 